\title{
Highly Tunable NEMS Shallow Arches
}

\author{
Syed N. R. Kazmi, Amal Z. Hajjaj, Pedro M. F. J. Costa, and Mohammad I. Younis*
}

\begin{abstract}
We report highly tunable nanoelectromechanical systems NEMS shallow arches under dc excitation voltages. Silicon based in-plane doubly clamped bridges, slightly curved as shallow arches, are fabricated using standard electron beam lithography and surface nanomachining of a highly conductive device layer on a silicon-on-insulator wafer. By designing the structures to have gap to thickness ratio of more than four, the mid-plane stretching of the nano arches is maximized such that an increase in the dc bias voltage will result into continuous increase in the resonance frequency of the resonators to wide ranges. This is confirmed analytically based on a nonlinear beam model. The experimental results are found to be in good agreement with that of the results from developed analytical model. A maximum tunability of $108.14 \%$ for a $180 \mathrm{~nm}$ thick arch with an initially designed gap of $1 \mu \mathrm{m}$ between the beam and the driving/sensing electrodes is achieved. Furthermore, a tunable narrow bandpass filter is demonstrated, which opens up opportunities for designing such structures as filtering elements in high frequency ranges.
\end{abstract}

\section{INTRODUCTION}

Micro/Nanoelectromechanical systems (MEMS/NEMS) are widely used as mass/gas sensors [1-4], memory element $[5,6]$, logic device $[7,8]$, energy harvester $[9,10]$, signal processing element [11], filtering and communication components $[12,13]$. Tunability of MEMS/NEMS structures is an extremely demanding feature for their use in modern communication systems to have on-demand frequency selection for various applications.

Several approaches have been presented to tune the resonance frequency of MEMS resonators by controlling the stiffness of the MEMS resonators with applied axial loads. Many approaches have been investigated to apply axial loads and tune the resonance frequency of a resonator, such as electrostatic [14], electromagnetic [15], and electrothermal [16]. A recent study has demonstrated large tunability of MEMS clamped-clamped straight micro beam resonators by controlling its axial load electrostatically [14] and electrothermally [16].

Since the early work of Nathanson [17], many researches have focused on the tunability of MEMS resonators by electrostatic means with applied dc bias voltage. One major drawback of the electrostatic tuning lies in its limitation of pull-in instability that leads to the collapse of the resonating structure with the stationary electrode especially when a relatively higher dc voltage is applied [18]. Typically, the electrostatic force is used to induce the softening effect in straight clamped-clamped beam that results a decrease in the

* Research supported by King Abdullah University of Science and Technology (KAUST).

Syed N. R. Kazmi, Amal Z. Hajjaj, Pedro M. F. J. Costa, and Mohammad. I. Younis are with, PSE Division, KAUST, Thuwal, Saudi Arabia (phone:+966128080597, e-mail: mohammad.younis@kaust.edu.sa ). resonance frequency of the MEMS structure [19], except few studies where they show that the resonance frequency could be tuned for higher values while increasing the dc voltage [20]. Sazonova et al. [21] demonstrated experimentally the tunability (increase) of the resonance frequencies at nano scale for Carbon Nano Tube (CNT) resonators by increasing the dc bias voltage, which is considered a distinct feature of CNTs due to their high stiffness.

In this current work, we present an investigation into the tunability of NEMS shallow arches and study the effect of geometric parameters, mainly thickness of the arch and the transduction gap between the beam and the stationary electrodes under dc bias voltages. Further, These silicon based shallow arches (either due to fabrication imperfection or intentionally fabricated as shallow arches can be used as basic building blocks for tunable band pass filters for high frequency applications.

\section{Materials AND MethodS}

\section{A. Problem Formulation}

The device under consideration, Fig. 1, consists of an initially curved clamped-clamped nano arch which is curved either due to the residual stresses from the fabrication process or designed to have such shape, actuated by a dc polarization voltage $V_{\mathrm{dc}}$ and an ac harmonic voltage of amplitude $V_{\mathrm{ac}}$ and frequency $\hat{\Omega}$, and is subjected to a viscous damping of coefficient $\hat{c}$. The initial shape $\hat{w}_{0}(x)$ is assumed to be of the buckled form [22].

$$
\hat{w}_{O}(\hat{x})=-\frac{1}{2} \hat{b}_{O}\left(1-\cos \left(2 \pi \frac{\hat{x}}{l}\right)\right)
$$

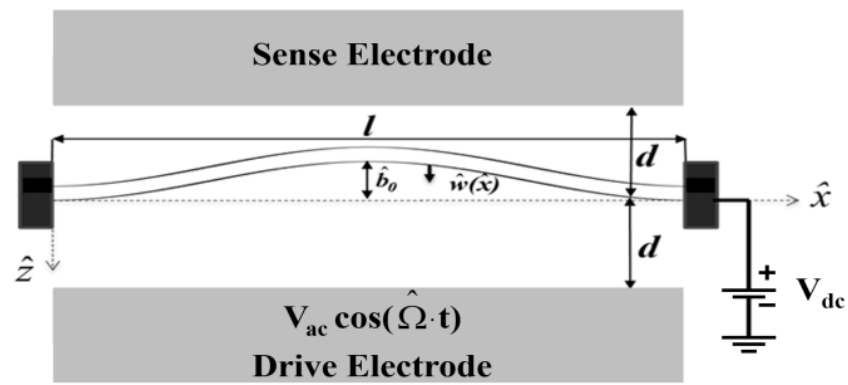

Fig. 1. Schematic of an electrostatically actuated clamped-clamped shallow arch.

The nonlinear Euler-Bernoulli equation of motion governing the transverse deflection of the arch $\hat{w}(\hat{x}, \hat{t})$ is written as follows [22-23]: 


$$
\begin{aligned}
& \rho b h \frac{\partial^{2} \hat{w}}{\partial \hat{t}^{2}}+\hat{c} \frac{\partial \hat{w}}{\partial \hat{t}}+E I_{s} \frac{\partial^{4} \hat{w}}{\partial \hat{x}^{4}}= \\
& \left(\frac{\partial^{2} \hat{w}}{\partial \hat{x}^{2}}+\frac{d^{2} \hat{w}_{0}}{d \hat{x}^{2}}\right)\left[\hat{N}+\frac{E A}{2 l} \int_{0}^{l}\left[\left(\frac{\partial \hat{w}}{\partial \hat{x}}\right)^{2}+2 \frac{\partial \hat{w}}{\partial \hat{x}} \frac{d \hat{w}_{0}}{d \hat{x}}\right) d \hat{x}\right] \\
& +\frac{1}{2} \varepsilon b \frac{\left(V_{d c}-V_{a c} \cos (\hat{\Omega} \cdot t)\right)^{2}}{\left(d-\hat{w}-\hat{w}_{0}\right)^{2}}-\frac{1}{2} \varepsilon b \frac{V_{d c^{2}}}{\left(d+\hat{w}+\hat{w}_{0}\right)^{2}}
\end{aligned}
$$

where $\hat{x}$ is the position along the nano arch, $\hat{t}$ is time, $l, b$ and $h$ are, respectively, the length, depth, and thickness of the nano arch, $\rho$ is the material density, $I_{s}=b h^{3} / 12$ is the moment of inertia of the rectangular cross-section of area $A=b h, E$ is Young's modulus, $d$ is the gap width between the nano arch and the stationary electrode, and $\varepsilon$ is the dielectric constant of the medium. $\hat{N}$ denotes the residual stress originated from the fabrication process. The last term represents the electrostatic force from the sensing electrode.

The nano arch is subjected to following boundary conditions:

$$
\hat{w}(0, \hat{t})=\hat{w}(l, \hat{t})=0 \text { and }\left.\frac{\partial \hat{w}}{\partial \hat{x}}\right|_{(0, \hat{t})}=\left.\frac{\partial \hat{w}}{\partial \hat{x}}\right|_{(l, \hat{t})}=0
$$

For convenience, we introduce the non-dimensional variables as below

$$
\begin{aligned}
& w=\frac{\hat{w}}{d} ; x=\frac{\hat{x}}{l} ; t=\frac{\hat{t}}{T} ; \\
& w_{0}=\frac{\hat{w_{0}}}{d}=\frac{1}{2} b_{0}(1-\cos (2 \pi x)) \text { and } b_{0}=\frac{\hat{b_{0}}}{d}
\end{aligned}
$$

where $T=\sqrt{\rho b h l^{4} / E I}$ is a time scale. Substituting (4) into (2) and (3), we obtain the non-dimensional equation of motion of the beam.

$$
\begin{aligned}
& \frac{\partial^{2} w}{\partial t^{2}}+c \frac{\partial w}{\partial t}+\frac{\partial^{4} w}{\partial x^{4}}= \\
& \left(\frac{\partial^{2} w}{\partial x^{2}}+\frac{d^{2} w_{0}}{d x^{2}}\right)\left[N+\alpha_{1} \int_{0}^{1}\left(\left(\frac{\partial w}{\partial x}\right)^{2}+2 \frac{\partial w}{\partial x} \frac{d w_{0}}{d x}\right) d x\right] \\
& +\alpha_{2} \frac{\left(V_{d c}-V_{a c} \cos (\Omega t)\right)^{2}}{\left(1-w-w_{0}\right)^{2}}-\alpha_{2} \frac{V_{d c}^{2}}{\left(1+w+w_{0}\right)^{2}}
\end{aligned}
$$

subject to the non-dimensional boundary conditions

$$
w(0, t)=w(1, t)=0 \text { and }\left.\frac{\partial w}{\partial x}\right|_{(0, t)}=\left.\frac{\partial w}{\partial x}\right|_{(1, t)}=0
$$

The non-dimensional (except for $\alpha_{2}$ ) parameters appearing in (5) are defined as below

$$
\alpha_{1}=6\left(\frac{d}{h}\right)^{2} ; \alpha_{2}=\frac{6 \varepsilon l^{4}}{E h^{3} d^{3}} ; N=\frac{l^{2}}{E I} \hat{N} ; c=\frac{l^{4}}{E I_{s} T} \hat{c} \text { and } \Omega=T \hat{\Omega}
$$

To solve the eigenvalue problem with the dc bias, the deflection of the nano arch under the electrostatic force is split into a static deflection, $w_{\mathrm{s}}(x)$, due to $V_{\mathrm{dc}}$ and a small dynamic deflection, $w_{\mathrm{d}}(x)$, as below

$w(x, t)=w_{s}(x)+w_{d}(x, t)$

The eigenvalue problem governing the variation of the resonance frequency with the dc bias is given by equation (9) assuming the Galerkin discretization [22].

$$
\begin{aligned}
\ddot{q}_{j} & =\left[2 \alpha_{1} \int_{0}^{l} \kappa_{j}\left(\frac{d^{2} w_{s}}{d x^{2}}+\frac{d^{2} w_{0}}{d x^{2}}\right) d x \int_{0}^{l}\left(\sum_{i=0}^{n} q_{i} \kappa_{i}\left(\frac{d w_{s}}{d x}+\frac{d w_{0}}{d x}\right)\right) d x\right] \\
& +\left[N+\alpha_{1} \int_{0}^{l}\left\{\left(\frac{d w_{s}}{d x}\right)^{2}+2\left(\frac{d w_{s}}{d x}\right)\left(\frac{d w_{0}}{d x}\right)\right\} d x\right] \int_{0}^{l} \kappa_{j}\left(\sum_{i=0}^{n} q_{i} \kappa_{i}{ }^{\prime \prime}\right) d x \\
& +\int_{0}^{l} \kappa_{j} \frac{2 \alpha_{2} V_{d c}{ }^{2}}{\left(1-w_{s}-w_{0}\right)^{3}}\left(\sum_{i=0}^{n} q_{i} \kappa_{i}\right) d x+\int_{0}^{l} \kappa_{j} \frac{2 \alpha_{2} V_{d c}{ }^{2}}{\left(1+w_{s}+w_{0}\right)^{3}}\left(\sum_{i=0}^{n} q_{i} \kappa_{i}\right) d x
\end{aligned}
$$

where $q_{i}(t)(i=0,1,2 . . n)$ denotes the non-dimensional modal coordinates and $\kappa_{i}(x)(i=0,1,2 . . n)$ denotes the unactuated shallow arch [22].Then, we compute the Jacobin of the system (equation (9)) for each value of $V_{d c}$ and find the corresponding resonance frequency by taking the square root of its eigenvalues [22].

One can note from (9) that the eigenvalue problem, and hence the resonance frequency of the resonators, depend strongly on the two parameters, $\alpha_{1}$ (non-dimensional) and $\alpha_{2}$ (dimensional), which are strong functions of thickness $h$ and gap $d$. By minimizing the $h$ and maximizing $d$, it is possible to strengthen the stretching of the bridge structure when applying a dc voltage while weakening the softening effect of the electrostatic force. Contrary to [20], using a perfectly straight beam between an equally distanced driving and sensing electrodes, the applied voltage between both electrodes and the beam cancel out the static deflection [23]. Thus the variation of the resonance frequency will be dominated by the softening effect of the electrostatic force.

\section{B. Fabrication and Measurements}

The shallow arches with gap to thickness ratio of more than four are fabricated from a <100> highly- conductive boron doped $\mathrm{Si}$ device layer of silicon-on-insulator (SOI) wafer. The fabrication process employ standard e-beam lithography and surface nanomachining techniques. The main fabrication steps are illustrated in Fig. 2. The fabrication starts with cleaning of SOI wafer by dipping it in a piranha solution, heated at $120{ }^{\circ} \mathrm{C}$, followed by a deionized (DI) water rinse to remove the organic residues. The native oxide is removed by using $1 \% \mathrm{HF}$ solution until the surface turns to hydrophobic followed by a deionized (DI) water rinse and spin drying, Fig. 2a. The thickness of the device layer is reduced from $2 \mu \mathrm{m}$ to $850 \mathrm{~nm}$ by thermal oxidation of the SOI wafer, Fig $2 \mathrm{~b}$, and then removing the thermally grown oxide using buffered oxide etch (BOE), Fig 2c. In order to achieve dimensions in nanometers range the e-beam lithography is employed to pattern 950 PMMA A4 positive tone e-beam resist, Fig. 2d. Reactive ion etching (RIE) is performed at $-10{ }^{\circ} \mathrm{C}$ to separate the nano beam, anchoring pads, and the RF electrodes from the rest of wafer surface. This entire wafer surface will serve as ground during the 
characterization of fabricated devices, Fig. 2e. Finally, the $\mathrm{HF}$ vapor etch is done to have free standing nano beams by removing the underneath $\sim 1 \mu \mathrm{m}$ box layer, Fig. $2 \mathrm{f}$. The beams are approximately $15 \mu \mathrm{m}$ long $(l), 850 \mathrm{~nm}$ deep $(b)$, $130 \mathrm{~nm}$ and $180 \mathrm{~nm}$ thick $(h)$ with $850 \mathrm{~nm}$ and $1 \mu \mathrm{m}$ transduction gaps (d) between the beam and the driving/sensing electrodes. Table I summarizes the dimensions of fabricated devices.

\begin{tabular}{|c|c|c|c|c|}
\hline & $\begin{array}{c}\text { Length } \\
(\mu \mathrm{m})\end{array}$ & $\begin{array}{c}\text { depth } \\
(\mathrm{nm})\end{array}$ & $\begin{array}{c}\text { Thickness } \\
(\mathrm{nm})\end{array}$ & $\begin{array}{c}\text { Gap } \\
(\boldsymbol{\mu} \mathrm{m})\end{array}$ \\
\hline D1 & 15 & 850 & 130 & 0.85 \\
\hline D2 & 15 & 850 & 130 & 1 \\
\hline D3 & 15 & 850 & 180 & 0.85 \\
\hline D4 & 15 & 850 & 180 & 1 \\
\hline
\end{tabular}

Table I. Designed dimensions for the fabricated nano devices.

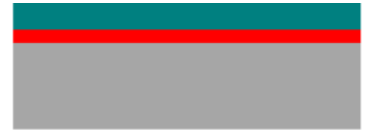

Substrate preparation

(a)

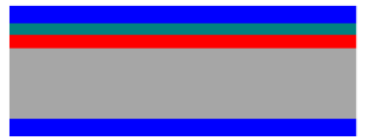

Thermal oxidation

(b)

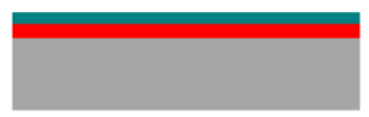

Buffered oxide etching

(c)

Thermally grown oxide

Handle wafer

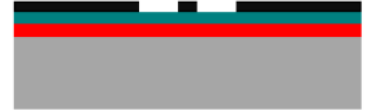

PMMA resist patterning

(d)

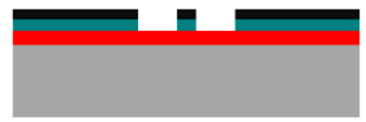

RIE of device layer

(e)

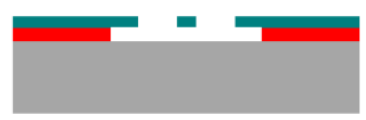

HF vapor etch

(f)

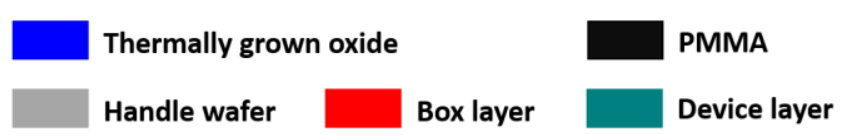

Fig. 2. Process flow for nano arches (a) Substrate cleaning and native oxide removal. (b) Thermal oxidation to thin down the device layer. (c) BOE treatment to remove the thermally grown oxide. (d) Patterning of e-beam resist (950 PMMA A4). (e) RIE to fabricate the nano arches and to separate the entire device structure from the ground plane. (f) HF vapor etching for free standing nano arches.

Fig. 3 shows an SEM image of a shallow arch fabricated using the fabrication process flow of Fig. 2. The beam is approximately $15 \mu \mathrm{m}$ long, $130 \mathrm{~nm}$ thick, and $850 \mathrm{~nm}$ deep with a transduction gap of $1 \mu \mathrm{m}$ between the arch and the driving/sensing electrodes. Note that the beam is slightly curved due to the residual stress in the device layer. Therefore, thinner beams are prone to relatively higher initial curvature than the thicker beams after HF vapor release. The observed initial rise ranges from $\sim 100 \mathrm{~nm}$ to $\sim 50 \mathrm{~nm}$ for beams of thickness $130 \mathrm{~nm}$ and $180 \mathrm{~nm}$, respectively.

The electrical characterization of the fabricated devices is performed in an ST-500 JANIS probe station, Fig. 4a, capable of measuring the arch response under controlled vacuum and varied chuck temperature conditions. The $\mathrm{S}_{21}$ transmission parameter, frequency response, is measured through Agilent E5071C network analyzer. Fig. 4b shows the photograph of the nano arch under test inside the probe station at room temperature.

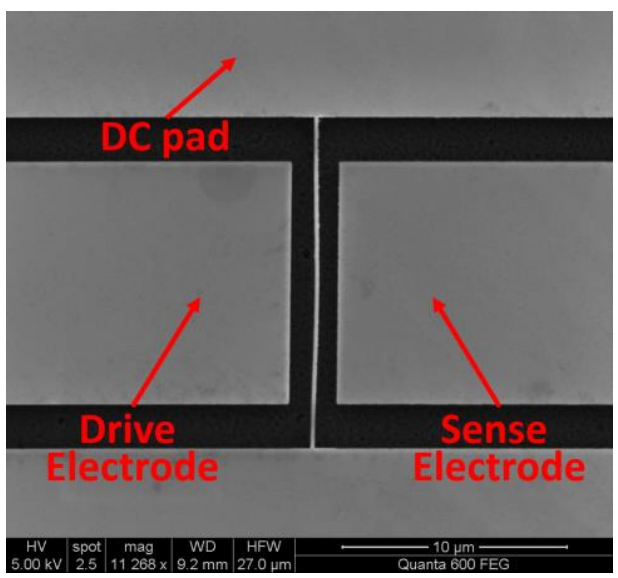

Fig. 3. An SEM image of an arch having a length (1) of $15 \mu \mathrm{m}$, depth (b) of $850 \mathrm{~nm}$, and thickness (h) of $130 \mathrm{~nm}$ with a transduction gaps (d) of $1 \mu \mathrm{m}$ between the beam and the driving/sensing electrodes.

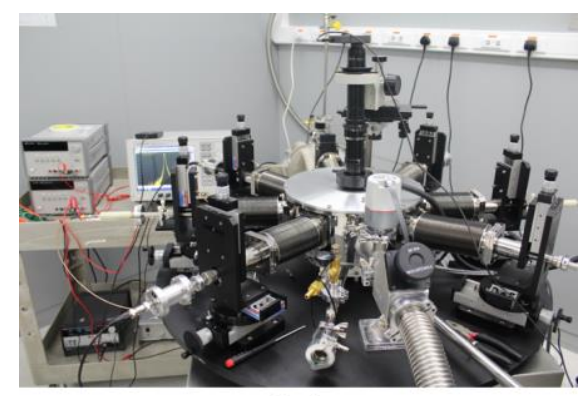

(a)

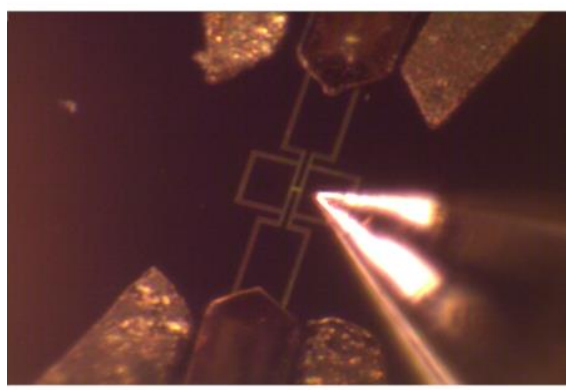

(b)

Fig. 4. (a) Photograph of ST-500 JANIS probe station having 2 RF and $4 \mathrm{dc}$ probe arms. (b) Snapshot of a nano arch under test.

Fig. 5 shows schematic of the driving and sensing configuration of the resonator in its two-port. The arch is excited by applying a dc bias voltage on it and an ac signal from one of the port of the network analyzer (Agilent E5071C) to one of its electrodes, named as driving electrode. The motional current is sensed through the other electrode, named as sensing electrode, and is fed into a low-noise 
amplifier (LNA) with its output coupled to the other port of the network analyzer.

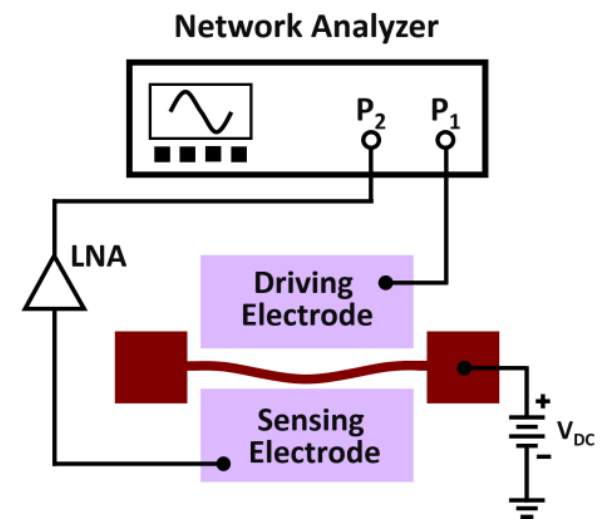

Fig. 5. A schematic of the measurement setup in its two-port driving and sensing configuration with dc polarization voltage applied to the nano arch.

\section{RESULTS AND DISCUSSION}

Fig. 6a and Fig. 6b show the variation of the resonance frequency of the $130 \mathrm{~nm}$ and $180 \mathrm{~nm}$ thick nano beams of 15 $\mu \mathrm{m}$ length, $850 \mathrm{~nm}$ depth, and with the initially designed transduction gaps of $850 \mathrm{~nm}$ and $1 \mu \mathrm{m}$, respectively, between the nano beam and the driving/sensing electrodes for various dc polarization voltages. The figures compare the analytical and experimental data. The analytical results are obtained by considering the effective values as in Table II, which are slightly different from the designed nominal values due to the fabrication tolerances.

\begin{tabular}{|c|c|c|c|c|c|}
\hline & $\begin{array}{c}\text { Length } \\
(\boldsymbol{\mu} \mathbf{m})\end{array}$ & $\begin{array}{c}\text { depth } \\
(\mathbf{n m})\end{array}$ & $\begin{array}{c}\text { Thickness } \\
(\mathbf{n m})\end{array}$ & $\begin{array}{c}\text { Gap } \\
(\mathbf{n m})\end{array}$ & $\begin{array}{c}\text { Initial rise } \\
(\mathbf{n m})\end{array}$ \\
\hline D1 & 15 & 850 & 70 & 850 & 122 \\
\hline D2 & 15 & 850 & 120 & 850 & 116 \\
\hline D3 & 15 & 850 & 70 & 1000 & 123 \\
\hline D4 & 15 & 850 & 120 & 1000 & 97.3 \\
\hline
\end{tabular}

Table II. Effective dimensions for obtaining the analytical results

The results show an increase in the resonance frequency for the beams with the increased dc bias voltages. The beams of $130 \mathrm{~nm}$ thickness with $850 \mathrm{~nm}$ and $1 \mu \mathrm{m}$ transduction gap between the beam and the driving/sensing electrodes show tunability of $77.12 \%$ and $85.51 \%$ under dc biased conditions, respectively. Similarly, the resonance frequencies of arches of $180 \mathrm{~nm}$ thickness are also found to increase with increasing the dc polarization voltages with tunability of $73.05 \%$ and $108.14 \%$ for $850 \mathrm{~nm}$ and $1 \mu \mathrm{m}$ transduction gap between the beam and the driving/sensing electrodes, respectively. A closer look towards the tunability of these beams reveals that those with $1 \mu \mathrm{m}$ transduction gaps have higher $\%$ tunability per volt, i.e., $66.07 \%$ and $45.14 \%$ compared to similar beams with $850 \mathrm{~nm}$ transduction gaps, i.e., $56.29 \%$ and $34.71 \%$ for dc bias changes from $40 \mathrm{~V}$ to $110 \mathrm{~V}$, respectively. This is clearly attributed to the dominating combined effect of thickness to gap ratio and the presence of initial curvature of the nano beam over the softening effect of the electrostatic force for large gaps. Moreover, thicker arches can achieve higher tunability compared to the thin arches due to the relatively higher pullin voltages. However, the thinner beams show higher tunability per unit increase in the dc polarization voltage due to the combined effect of increasing arch stiffness and dominating effect of mid-plane stretching over electrostatic force for large gaps.

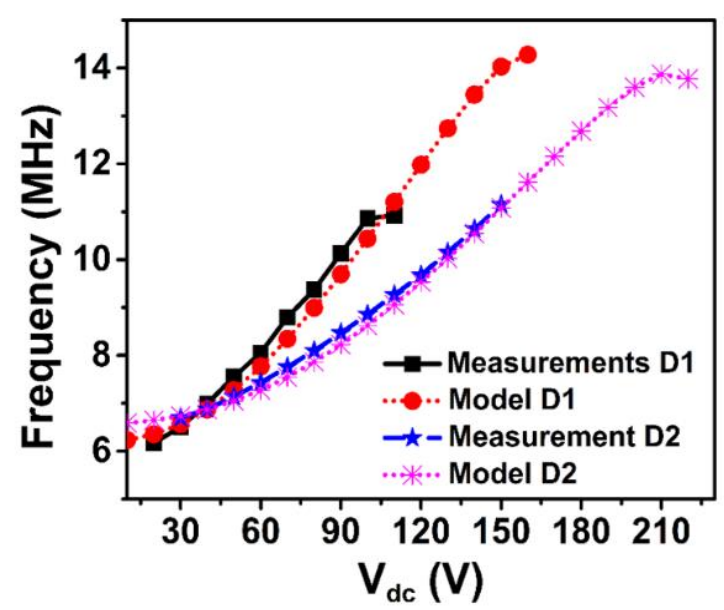

(a)

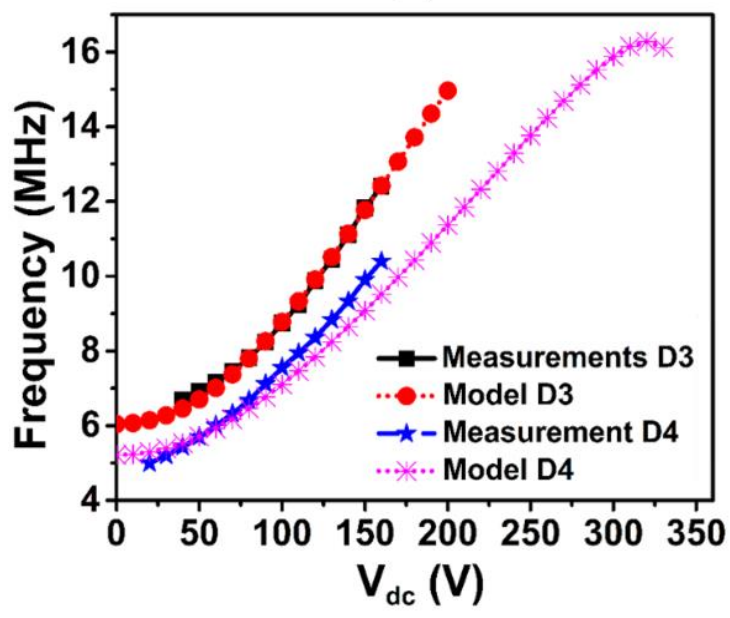

(b)

Fig. 6. The dc voltage versus resonance frequency for the beams of $15 \mu \mathrm{m}$ length, $850 \mathrm{~nm}$ depth, and (a) $130 \mathrm{~nm}$ thickness with a transduction gap of $850 \mathrm{~nm}$ and $1 \mu \mathrm{m}$, respectively. (b) $180 \mathrm{~nm}$ thickness with a transduction gap of $850 \mathrm{~nm}$ and $1 \mu \mathrm{m}$, respectively. In (a) and (b), simulations results are compared with the experimental data.

Next, we demonstrate using such tunability for filtering applications. Fig. 7a shows a schematic for the measurement setup for two near-identical electrically coupled resonators for a narrow bandpass filter demonstration. The two resonators are biased in such a way that the first dc source results in equal voltage on the beams. A second dc source is used to tune the resonance frequency of the resonators with lower resonance frequency and to bring it closer to the resonance frequency of other resonator. This slight difference in resonance frequency of the two adjacent resonators is due to fabrication tolerances. The input RF signal from port 1 of the network analyzer is fed into input electrodes of the two resonators through a splitter. The output is received at a common sensing electrode and directly fed into LNA with its 
output coupled to the other port of the network analyzer. Fig. $7 \mathrm{~b}$ shows an SEM image of the fabricated structure for bandpass filtering application.

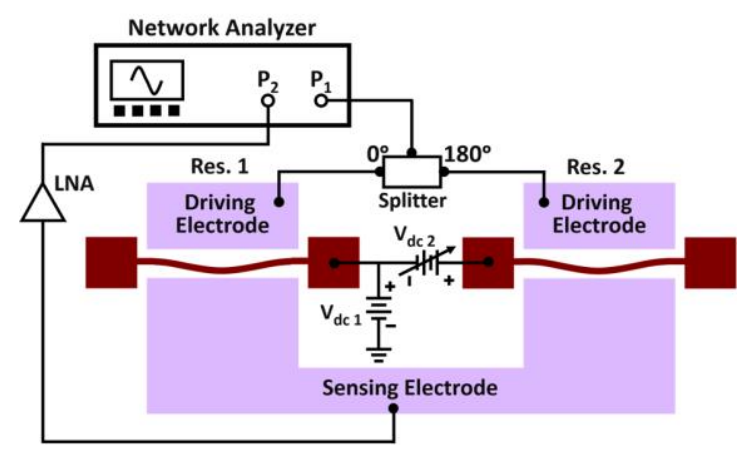

(a)

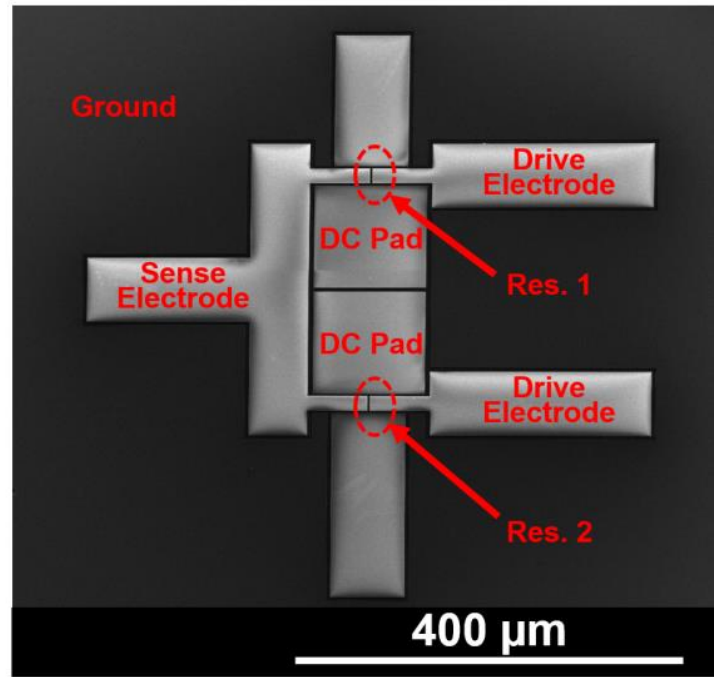

(b)

Fig. 7. (a) Schematic of the driving and sensing configuration for the electrically coupled nanomechanical shallow arch beams under dc bias conditions for narrow bandpass filtering. (b) An SEM image showing the layout for electrically coupled nanomechanical arches of $15 \mu \mathrm{m}$ length, $850 \mathrm{~nm}$ depth, $180 \mathrm{~nm}$ thickness, and $1 \mu \mathrm{m}$ transduction gaps between the beam and the driving/sensing electrodes for filtering.

Fig. 8a shows the magnitude of $\mathrm{S}_{21}$ transmission parameters (response in black) for electrically coupled resonators showing resonance peaks at 5.186 MHz and 5.147 $\mathrm{MHz}$ for a dc bias voltage of $40 \mathrm{~V}$ and input RF power of 6.3 $\mathrm{dBm}$ from port 1 of the network analyzer. Resonator 2 is tuned by applying an additional dc voltage so that both resonance peaks come closer (response in red) to realize a narrow bandpass filter with a center frequency of $5.175 \mathrm{MHz}$ and a $3 \mathrm{~dB}$ band width of $\sim 33 \mathrm{kHz}$, as in Fig. 8b. The filter's center frequency can be tuned to $5.446 \mathrm{MHz}$ with a $3 \mathrm{~dB}$ band width of $\sim 19 \mathrm{kHz}$ (response in blue) by increasing the $\mathrm{dc}$ bias voltage to $50 \mathrm{~V}$ and tuning the frequency of second resonators to achieve narrow band filer response, as in Fig. $8 \mathrm{~b}$. The practical realization of tunable band pass filters using electrically coupled nano arches with the flexibility of adjustable bandwidth can open up the possibilities for TV tuner applications. Higher frequency filtering can be achieved by shrinking the device dimensions with similar designs with higher Young's modulus to density ratio materials than silicon like silicon carbide [24].

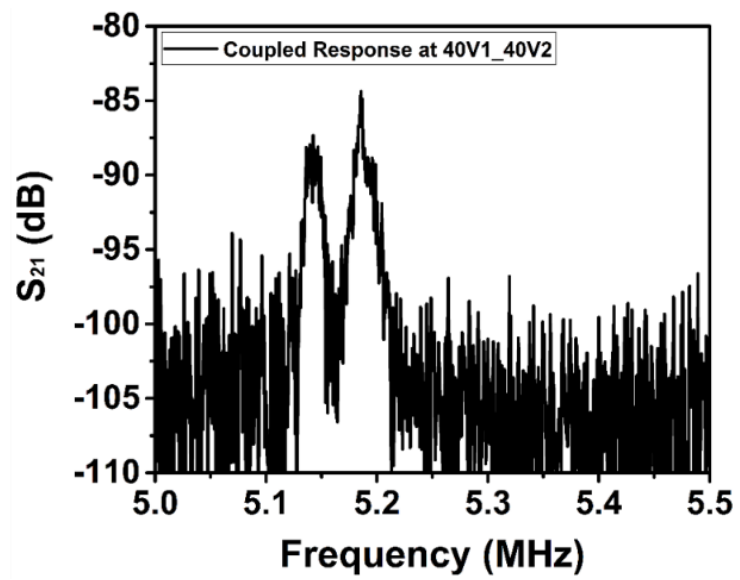

(a)

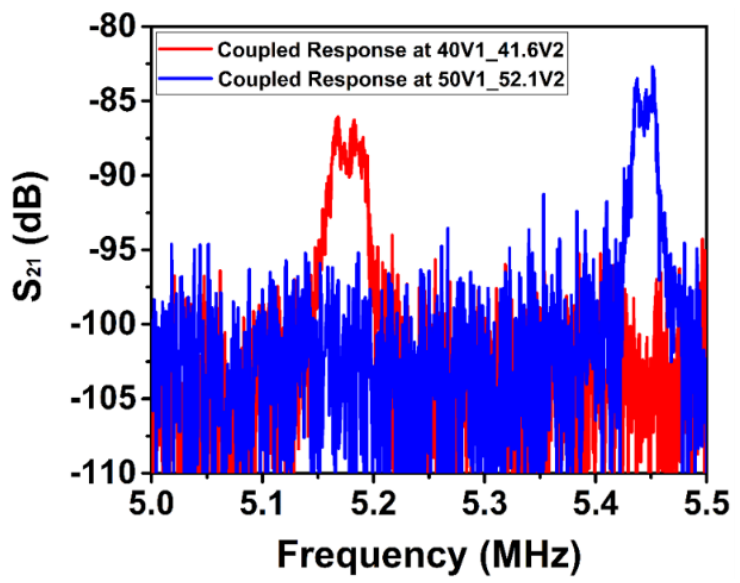

(b)

Fig. 8. (a) The magnitude of $S_{21}$ transmission parameter for two electrically coupled nano beams at dc bias of $40 \mathrm{~V}$ and $6.3 \mathrm{dBm} \mathrm{RF}$ power. (b) The magnitude of S21 transmission parameter for the electrically coupled beams demonstrating a tunable narrow bandpass filter in high frequency range under dc polarization voltages of 40 $\mathrm{Vdc} 1$ and $50 \mathrm{Vdc} 1$ (on arch 1) and $41.6 \mathrm{Vdc} 2$ and $52.1 \mathrm{Vdc} 2$ (on arch 2) at $6.3 \mathrm{dBm}$ input RF power.

\section{CONCLUSION}

In conclusions, an analytical and experimental study is presented on the tunability of in-plane doubly-clamped imperfect nanomechanical beams under varied dc bias conditions at room temperature. The experimental results showed good agreement with that of the analytical ones for the beams with carefully designed gap to thickness ratios. The beams show high tunability by electrostatic actuation due to dominant mid-plane stretching over softening effect of electrostatic force. This mid-plane stretching effect is due to the combined effect of the thickness to gap ratio and the initial imperfection of the nano beams. A tunability of up to 
$108.14 \%$ for a carefully designed shallow arch is experimentally demonstrated. Moreover, a tunable narrow bandpass filter is also presented using the highly tunable nano arches. The high tunability of these arches opened up the possibilities for tunable band pass filtering in high frequency range (3-30 MHz).

\section{ACKNOWLEDGMENT}

This work was supported by King Abdullah University of Science and Technology (KAUST) research funds.

\section{REFERENCES}

[1] K. Park, N. Kim, D. T. Morisette, N. Aluru, R. Bashir, "Resonant MEMS mass sensors for measurement of microdroplet evaporation", J. Microelectromech. Syst., Vol. 21(3), pp. 702-711, (2012).

[2] T. P. Burg, A. R. Mirza, N. Milovic, C. H. Tsau, G. Popescu, J. S. Foster, S. R. Manalis, "Vacuum-packaged suspended microchannel resonant mass sensor for biomolecular detection", $J$. Microelectromech. Syst., Vol. 15(6), pp. 1466-1476, (2006).

[3] E. Sage, A. Brenac, T. Alava, R. Morel, C. Dupré, M. S. Hanay, M. L. Roukes, L. Duraffourg, C. Masselon, and S. Hentz, "Neutral particle mass spectrometry with nanomechanical systems", Nat. Commun., Vol. 6, pp. 6482, (2015).

[4] I. Bargatin, E. B. Myers, J. S. Aldridge, C. Marcoux, P. Brianceau, L. Duraffourg, E. Colinet, S. Hentz, P. Andreucci, and M. L. Roukes. "Large-scale integration of nanoelectro-mechanical systems for gas sensing applications", Nano Lett., Vol. 12, pp. 1269-1274, (2012).

[5] M. A. Al Hafiz, L. Kosuru, and M. I. Younis, "Electro-thermal Frequency Modulated Resonator for Mechanical Memory", $J$. Microelectromech. Syst., Vol. 25(5), pp. 877-883, (2016).

[6] T. Rueckes, K. Kim, E. Joselevich, G. Y. Tseng, C. Cheung and C. M. Lieber, "Carbon nanotube-based nonvolatile random access memory for molecular computing", Science, Vol. 289, pp. 94-97, (2007).

[7] M. A. Al Hafiz, L. Kosuru, and M. I. Younis, "Microelectromechanical reprogrammable logic device", Nat. Commun., Vol. 7, (2016).

[8] S. N. R. Kazmi, M. A. Al Hafiz, K. C. Nanaiah, S. Ilyas, J. Holguin, P. M. F. J. Costa and M. I. Younis, "Tunable nanoelectromechanical resonator for logic computations", Nanoscale, Vol. 9, pp. 3449-3457, (2017).

[9] G. Altena, D. Hohlfeld, R. Elfrink, M. H. Goedbloed, and R. van Schaijk, "Design, modeling, fabrication and characterization of an electret-based MEMS electrostatic energy harvester", Int. Solid-State Sens., Actuators and Microsyst. Conf., (2011).

[10] Q. Shi, T. Wang and C. Lee, "MEMS based broadband piezoelectric ultrasonic energy harvester (PUEH) for enabling self-powered implantable biomedical devices", Sci. Rep., Vol. 6, pp. 24946, (2016).

[11] L. Lin, R. T. Howe, and A. P. Pisano, "Microelectro-mechanical filters for signal processing", J. Microelectromech. Syst., Vol. 7(3), pp. 286294, (1998).

[12] J. N. Nilchi, R. Liu, and C. T.-C. Nguyen, "7th order sharp-roll-off bridged micromechanical filter", Int. Solid-State Sens., Actuators and Microsyst. Conf., (2015).

[13] C. T-C. Nguyen, "Frequency-selective MEMS for miniaturized lowpower communication devices", IEEE Trans. Microw. Theory Tech., Vol. 47(8), pp. 1486-1503, (1999).

[14] E. M. Abdel-Rahman, M. I. Younis, and A. H. Nayfeh, "Characterization of the mechanical behavior of an electrically actuated microbeam", J. Micromech. Microeng., Vol. 12(6), pp. 759, (2002).

[15] H. Maekoba, P. Helin, G. Reyne, T. Bourouina, and H. Fujita, "Selfaligned vertical mirror and V-grooves applied to an optical-switch: modeling and optimization of bi-stable operation by electromagnetic actuation", Sens. Actuators A Phys., Vol. 87(3), pp. 172-178, (2001).

[16] A. Z. Hajjaj, N. Alcheikh, A. Ramini, M. A. Al Hafiz, and M. I. Younis, "Highly tunable electrothermally and electrostatically actuated resonators", J. Microelectromech. Syst., Vol. 25(3), pp. 440449, (2016).
[17] H. C. Nathanson, W. E. Newell, R. A. Wickstrom, and J. R. Davis, "The resonant gate transistor", IEEE Trans. Electron Dev., Vol. 14(3), pp. 117-133, (1967).

[18] M. I. Younis, R. Miles, and D. Jordy, "Investigation of the response of microstructures under the combined effect of mechanical shock and electrostatic forces", J. Micromech. Microeng., Vol. 16(11), pp. 2463, (2006).

[19] A. H. Nayfeh, M. I. Younis, and E. M. Abdel-Rahman, "Reducedorder models for MEMS applications", Nonlinear dyn., Vol. 41(1), pp. 211-236, (2005).

[20] A. Z. Hajjaj, A. Ramini, and M. I. Younis, "Experimental and analytical study of highly tunable electrostatically actuated resonant beams", J. Micromech. Microeng., Vol. 25(12), pp. 125015, (2015).

[21] V. Sazonova, Y. Yaish, H. Üstünel, D. Roundy, T. A. Arias, and P. L. McEuen, "A tunable carbon nanotube electromechanical oscillator", Nature, Vol. 431, pp. 284-287, (2004).

[22] M. I. Younis, "MEMS Linear and Nonlinear Statics and Dynamics", Vol. 20. New York, NY, USA: Springer, (2011).

[23] N. Kacem and S. Hentz, "Bifurcation topology tuning of a mixed behavior in nonlinear micromechanical resonators", Appl. Phys. Lett., Vol. 95, pp. 183104, (2009).

[24] X. M. H. Huang, C. A. Zorman, M. Mehregany and M. L. Roukes, "Nanoelectromechanical systems: Nanodevice motion at microwave frequencies", Nature, Vol. 421, pp. 496-496, (2003). 